\title{
Peripheral blood lymphocyte-to-monocyte ratio as a prognostic factor in advanced epithelial ovarian cancer: a multicenter retrospective study
}

\author{
Jia-yu Zhu ${ }^{1 *}$, Cheng-cheng Liu $^{2 *}$, Liang Wang 3 , Mei Zhong1, Hai-lin Tang2 ${ }^{2}$, Hua Wang ${ }^{3} \bowtie$ \\ 1. Department of Obstetrics and Gynecology, Nanfang Hospital of Southern Medical University, Guangzhou 510515, P.R. China; \\ 2. Sun Yat-sen University Cancer Center, State Key Laboratory of Oncology in South China; Collaborative Innovation Centre for Cancer Medicine, Guangzhou \\ 510060, P. R. China; \\ 3. Sun Yat-sen University Cancer Center, State Key Laboratory of Oncology in South China; Collaborative Innovation Centre for Cancer Medicine, Guangzhou \\ 510060, P. R. China; Department of Hematological Oncology, Sun Yat-Sen University Cancer Center, Guangzhou 510060, P. R. China. \\ * Jia-yu Zhu, Cheng-cheng Liu and Liang Wang contributed equally to this article.
}

$\square$ Corresponding authors: Hua Wang, Department of Hematological Oncology, Sun Yat-sen University Cancer Center; State Key Laboratory of Oncology in South China; Collaborative Innovation Center for Cancer Medicine, Guangzhou, 510060, P.R. China E-mail: wanghua@sysucc.org.cn; Phone No.:+86-20-87342462 or Hailin Tang, Sun Yat-sen University Cancer Center, State Key Laboratory of Oncology in South China; Collaborative Innovation Center for Cancer Medicine, Guangzhou, 510060, P.R. China E-mail: tanghl@sysucc.org.cn; Phone No..:+86-20-87342438.

(c) Ivyspring International Publisher. This is an open access article distributed under the terms of the Creative Commons Attribution (CC BY-NC) license (https://creativecommons.org/licenses/by-nc/4.0/). See http://ivyspring.com/terms for full terms and conditions.

Received: 2016.09.22; Accepted: 2016.12.22; Published: 2017.02.25

\begin{abstract}
The lymphocyte-to-monocyte ratio (LMR), as a surrogate marker of systemic inflammation, has been found to be a novel prognostic indicator in various malignancies. Data from 672 advanced epithelial ovarian cancer (EOC) patients treated with neoadjuvant chemotherapy (NAC) followed by debulking surgery were analyzed, and the prognostic value of $L M R$ were evaluated. The optimal cutoff point of LMR in prediction of survival was defined as 3.45 through receiver operating characteristics curve analysis. Patients with low LMR $(\leq 3.45)$ at diagnosis tended to have more adverse clinical features, such as higher histological grade, chemotherapy resistance, and residual tumor $>1 \mathrm{~cm}$ after debulking surgery. No significant correlation was found between LMR level and age and histological type. Moreover, after NAC, the complete remission (CR) rate for the low-LMR group was lower than those for the high-LMR group $(P<0.05)$. Patients with low $L M R$ had poorer progression-free survival (PFS; $P<0.001)$ and overall survival $(O S ; P<0.001)$. Multivariate analysis revealed that low LMR was an independent adverse predictor for PFS and OS. Results indicated that low LMR at diagnosis is a novel independent prognostic factor for advanced EOC. However, prospective study is needed to validate this prognostic factor and biological studies should further investigate the mechanisms underlying the correlation between low LMR and poor prognosis in advanced EOC.
\end{abstract}

Key words: lymphocyte-to-monocyteratio; epithelial ovarian cancer; neoadjuvant chemotherapy; prognosis

\section{Introduction}

Epithelial ovarian cancer (EOC) is the most predominant ovarian malignancy (approximately $90 \%$ ) [1] and is the leading cause of death from gynecologic malignant tumor [2]. Although cytoreductive surgery followed by platinum-based adjuvant chemotherapy significantly prolong survival period, the advanced EOC patients have only less than $40 \%$ of five-year survival rate [3]. To improve outcomes, neoadjuvant chemotherapy (NAC) combined with debulking surgery is often used as an alternative mode of treatment for patients with advance-stage EOC over the last decade [4]. However, chemotherapeutic resistance and relapse still frequently occurred, leading to poor response and prognosis. Clinicopathological factors, such as histologic differentiation degree, Federation 
International of Gynecology and Obstetrics (FIGO) stage and cancer antigen 125 (CA-125) have been proven to be predictive factors for survival in patients with EOC after a comprehensive therapy involving surgery and chemotherapy [5-7]. However, the capability of these conventional tumor parameters for predicting recurrence and survival is inadequate.

Recently, increasing evidence showed that the peripheral blood white cells, for instance lymphocytes and monocytes, are significantly related to prognosis in various cancers. Absolute lymphocyte count was proven to be an independent prognostic factor in bladder cancer, melanoma and extranodal natural killer/T-cell lymphoma [8-10]. Elevated monocyte count has been demonstrated to predict poor prognosis in mantle cell lymphoma, colorectal cancer and lung adenocarcinoma [11-13]. Lymphocyte-to-monocyte ratio (LMR) has been shown to be a prognostic factor for patients with hepatocellular carcinoma and endometrial cancer [14, 15]. Recently, one study showed that LMR predicts the survival and aggressiveness of malignancy of ovarian cancer patients who underwent primary debulking surgery [16]. However, few studies have evaluated the prognostic significance of LMR in advanced EOC patients treated with neoadjuvant chemotherapy (NAC) followed by debulking surgery. Hence, this study was performed to investigate the prognostic value of pretreatment LMR in these patients.

\section{Materials and methods}

\section{Patient selection}

A total of 672 patients newly diagnosed with EOC from Jun 2008 to Dec 2015 at the Sun Yat-Sen University Cancer Center and Nanfang Hospital of Southern Medical University were selected for this study. The initial diagnosis of EOC was conducted through a core needle biopsy before treatment. Meanwhile, histological grades were determined based on the World Health Organization classification standards. The inclusion criteria for patient selection whereas follows: (1) EOC was pathologically confirmed; (2) received NAC followed by debulking surgery; (3) sufficient clinical information and follow-up data.

Patient demographics, Eastern Cooperative Oncology Group performance status (ECOG PS), serum CA-125, peripheral blood cell counts, surgical outcomes (size of remaining tumor) and other clinical pathologic parameters were collected. Whole body computed tomography (CT) or positron emission tomography/computed tomography (PET/CT) was performed in each patient. For all patients, the tumor stage was determined for all patients according to the FIGO staging system. The LMR was calculated by dividing the absolute lymphocyte count with the absolute monocyte count, according to pretreatment peripheral blood cell count. The ethics committees of all the hospitals approved this study and informed consent for using their medical records for research purposes was obtained from all individual participants enrolled in this study.

\section{Treatment}

All patients received three cycles of preoperative chemotherapy. The NAC regimen comprised the following: (1) cisplatin/carboplatin combined with paclitaxel and (2) carboplatin plus docetaxel. The surgeries were performed three to six weeks after chemotherapy. We assessed chemotherapy responses according to the Response Evaluation Criteria in Solid Tumors (version 1.0) [17]. Complete response or partial response was considered as chemotherapy sensitivity, whereas stable disease or progressive disease was determined as chemotherapy resistance. After the complete treatment ends, follow-up visits were performed in the outpatient department once every three months for the first two years and then every six months or until death.

\section{Statistical analysis}

The relationship of LMR with clinicopathological characteristics of EOC was analyzed using chi-square tests. The receiver operating characteristics (ROC) curve analysis was used to determine the cutoff value for LMR predicting survival, by searching the maximum sensitivity and specificity point. The Kaplan-Meier method was used to calculate overall survival (OS) and progression-free survival (PFS). Meanwhile, the univariate analyses for evaluating the effects of clinicopathological features, including LMR, on survival were performed through log-rank test. The Cox proportional hazard model was used to conduct the multivariate analysis. A two-tailed $\mathrm{P}<0.05$ was defined statistically significant. The SPSS software (version 19.0) was utilized for statistical analysis.

\section{Results}

\section{Patient characteristics and correlation between LMR and clinical features}

A total of 672 patients, with a median age of 55, were enrolled in this study. The patient clinical characteristics are listed in Table 1. Pathology of the 672 ovarian cancer patients consisted of $484(72.0 \%)$ serous ovarian carcinoma cases and 188 (28.0\%) non-serous ovarian carcinoma cases. Majority of the cases (494 patients, $73.5 \%$ ) displayed a good 
performance status (ECOG PS 0-1). A total of 564 patients with EOC were categorized as FIGO stage III, and 108 were diagnosed with FIGO stage IV EOC. The baseline serum CA-125 level in 446 patients $(66.4 \%)$ was higher than normal $(35 \mathrm{U} / \mathrm{mL})$. After cytoreductive surgery, $72.3 \%(486 / 672)$ cases had residual tumor size less than $1 \mathrm{~cm}$.

Table 1.The clinicopathological characteristics of patients with EOC

\begin{tabular}{ll}
\hline Variables & $\mathbf{n}(\%)$ \\
\hline Age [median (range), years] & $55(30-70)$ \\
$<55$ & $318(47.3)$ \\
$\geq 55$ & $354(52.7)$ \\
ECOG PS & \\
$\leq 1$ & $494(73.5)$ \\
$>2$ & $178(26.5)$ \\
Histological type & \\
Serous & $484(72.0)$ \\
Non-serous & $188(28.0)$ \\
Histological grade & \\
G1 & $384(57.1)$ \\
G2/G3 & $288(42.9)$ \\
FIGO stage & \\
III & $564(83.9)$ \\
IV & $108(16.1)$ \\
CA-125 (U mL-1) & \\
$\leq 35$ & $226(33.6)$ \\
$>35$ & $446(66.4)$ \\
Chemosensitivity & \\
Yes & $444(66.1)$ \\
No & $228(33.9)$ \\
Residual tumor (cm) & \\
$\leq 1$ & $486(72.3)$ \\
$>1$ & $186(27.7)$ \\
Lymphocytes Count (10 9 /L) & $1.50(0.56-4.20)$ \\
Median value, (range) & \\
Monocytes Count (10 9 /L) & $0.47(0.10-1.74)$ \\
Median value, (range) & \\
Lymphocyte-to-monocyte ratio, LMR & $3.40(0.90-9.76)$ \\
Median value, (range) & \\
\hline Abbreviations: EOC, epithelial ovarian cancer; ECOG PS, Eastern Cooperative \\
Group performance status; G1, well differentiated; G2, moderately differentiated; \\
G3, poorly differentiated; FIGO, International Federation of Gynecology and \\
Obstetrics; CA -125, cancer antigen 125,LMR, lymphocyte-to-monocyte ratio. \\
\end{tabular}

The median values of peripheral blood lymphocyte and monocyte for all patients were $1.50 \times$ 109/L (range: 0.56-4.20) and $0.47 \times 10^{9} / \mathrm{L}$ (range: $0.10-1.74)$, respectively.

The best cutoff point of LMR in prediction of survival was determined to be 3.45 through ROC curve analysis, with an area under the curve value of $0.808(\mathrm{P}<0.001)$ (Fig 1). ROC analysis demonstrated that LMR was more specific for predicting survival than CA-125 level $(86.7 \%$ vs. $73.6 \%$, Supplementary Figure S1). Based on this cutoff value, 340 (50.6\%) patients were classified into the low-LMR group $(\leq 3.45)$, and 332 patients $(49.4 \%)$ were categorized into the high-LMR group (>3.45) at diagnosis. The baseline clinicopathological characteristics were compared between patients in low-LMR group and high-LMR group (Table 2). ECOG PS of patients was poorer in the low-LMR group than high-LMR group. Patients in the low-LMR group were more likely to have higher histological grade and higher CA-125 level and were characterized by more common chemotherapy resistance and more frequent postoperative residual tumor larger than $1 \mathrm{~cm}$. However, no significant differences were noted between low-LMR and high-LMR groups in age and histological type.

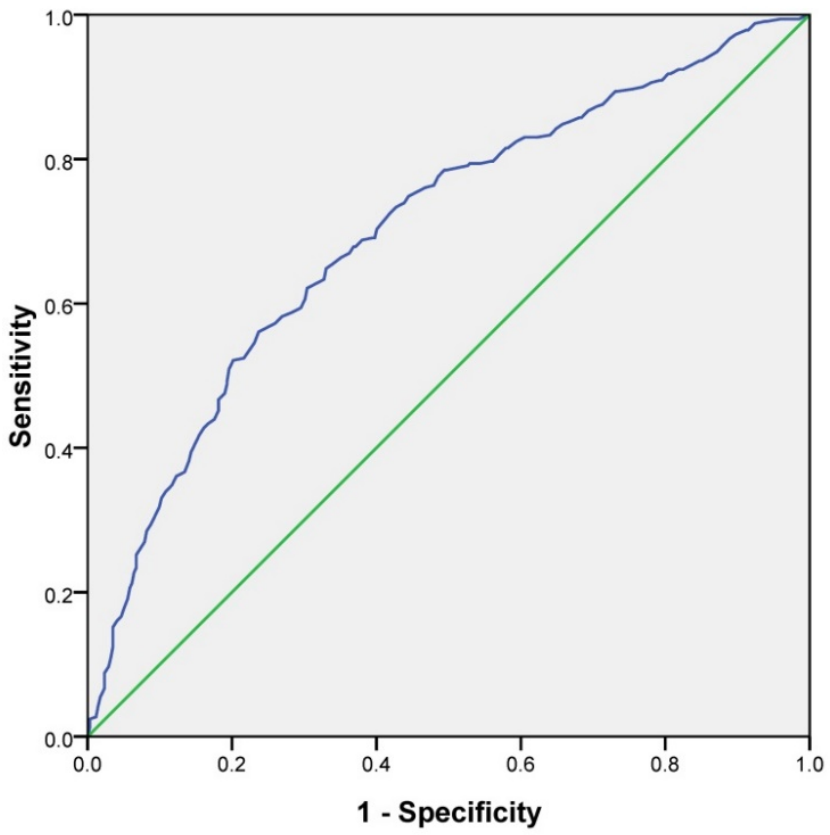

Figure 1. ROC curve analysis for the optimal cutoff point of LMR. The most discriminative cutoff value of LMR was 3.45 with an AUC value of 0.808 . The sensitivity and specificity were 73.4 and $73.0 \%$, respectively.

\section{NAC regimen and response}

In this study, all patients received NAC, of which 298 patients (44.3\%) were given cisplatin plus paclitaxel, 250 cases (37.2\%) received combined chemotherapy with paclitaxel and carboplatin, and 124 cases $(18.5 \%)$ received carboplatin and docetaxel combination chemotherapy regimen. Of the 672 EOC patients enrolled, 444 displayed chemotherapy sensitivity and 228 showed chemotherapy resistance. After the NAC, 61 patients $(9.1 \%)$ had complete remission (CR). The CR rate for NAC was significantly lower for patients in the low-LMR group compared with those in the high-LMR group $(\mathrm{P}<0.05)$.

\section{Survival and prognostic factors}

At a median follow-up time of 38 months (range, 5-103 months), the median PFS and OS for all 672 patients was 25.0 months (95\% CI 23.7-26.3 months) 
and 49.0 months (95\% CI 45.5 - 52.5 months), respectively. Patients in low-LMR group had significantly poorer PFS (median PFS: 22.0 months vs. 31.0 months, P<0.001; Fig.2a) and OS (median OS: 44.0 months vs. 58.0 months, $\mathrm{P}<0.001$; Fig.2b). In univariate analysis, high histological grade, advanced FIGO stage, high serum CA-125 level, chemotherapy resistance, residual tumor $>1 \mathrm{~cm}$, lower LMR value $(\leq$ $3.45)$, etc. were significantly associated with poorer PFS and OS (Table 3). No significant relationships were found between age, ECOG PS score and survival outcomes. As shown in Table 3, multivariate analysis using Cox regression model showed that lower LMR value $(\leq 3.45)$, residual tumor $>1 \mathrm{~cm}$, chemotherapy resistance and advanced FIGO stage were four independent prognostic factors for both PFS and OS $(\mathrm{P}<0.05)$.

\section{Discussion}

In the current study, lower LMR was found to be significantly correlated with poor ECOG PS, higher histological grade, higher CA-125 level, chemotherapy resistance and larger residual tumor after the debulking surgery in advanced EOC. Furthermore, lower LMR was associated with poor responses to NAC. Based on the Cox regression model, which included LMR, FIGO stage, serum CA-125 level, chemosensitivity, etc., LMR was found to be an independent prognostic factor for both PFS

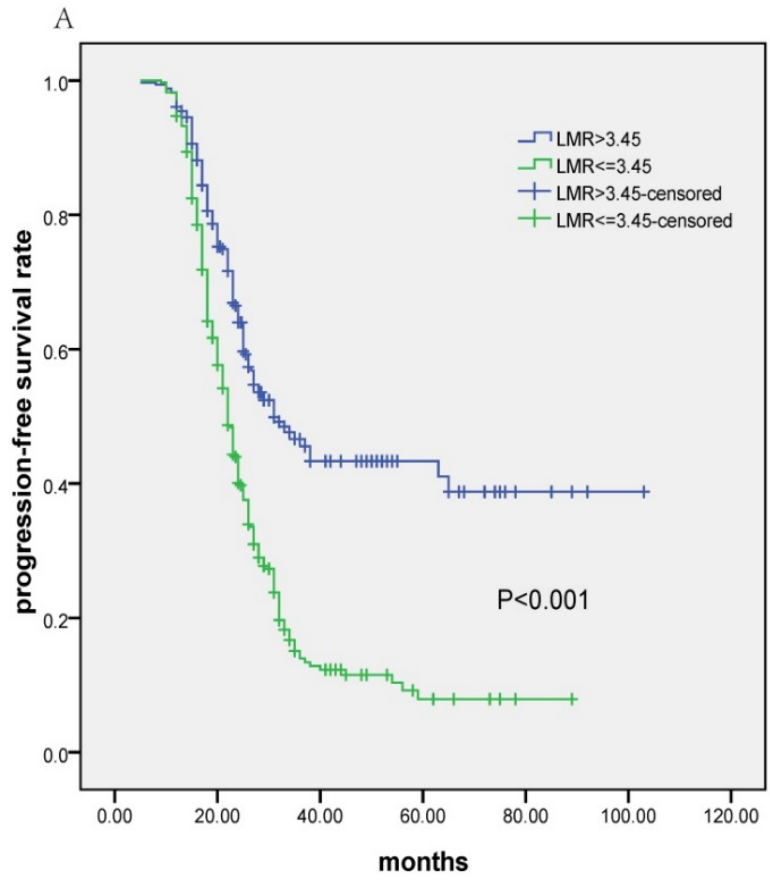

and OS.

Table 2. Correlation of LMR with clinicopathological features in patients with EOC

\begin{tabular}{|c|c|c|c|}
\hline Variables & $\begin{array}{l}\mathrm{LMR} \leq 3.45 \\
\mathrm{n}=340(\%)\end{array}$ & $\begin{array}{l}\text { LMR>3.45, } \\
n=332(\%)\end{array}$ & $P$ \\
\hline Age & & & 0.189 \\
\hline$<55$ & $152(44.7)$ & $166(50.0)$ & \\
\hline$\geq 55$ & $188(55.3)$ & $166(50.0)$ & \\
\hline ECOG PS & & & $<0.001$ \\
\hline $0-1$ & $214(62.9)$ & $280(84.3)$ & \\
\hline$\geq 2$ & $126(37.1)$ & $52(15.7)$ & \\
\hline Histological type & & & 0.732 \\
\hline Serous & $247(72.6)$ & $237(71.4)$ & \\
\hline Non-serous & $93(27.4)$ & $95(28.6)$ & \\
\hline Histological grade & & & 0.001 \\
\hline G1 & $173(50.9)$ & $211(63.6)$ & \\
\hline G2/G3 & $167(49.1)$ & $121(36.4)$ & \\
\hline FIGO stage & & & $<0.001$ \\
\hline III & $269(79.1)$ & 295(88.9) & \\
\hline IV & $71(20.9)$ & $37(11.1)$ & \\
\hline CA-125 (U mL-1) & & & $<0.001$ \\
\hline$\leq 35$ & $83(24.4)$ & $143(43.1)$ & \\
\hline$>35$ & $257(75.6)$ & $189(56.9)$ & \\
\hline Chemosensitivity & & & 0.001 \\
\hline Yes & $204(60.0)$ & $240(72.3)$ & \\
\hline No & $136(40.0)$ & $92(27.7)$ & \\
\hline Residual tumor (cm) & & & $<0.001$ \\
\hline$\leq 1$ & $204(60.0)$ & $282(84.9)$ & \\
\hline$>1$ & $136(40.0)$ & $50(15.1)$ & \\
\hline
\end{tabular}

Abbreviations: EOC, epithelial ovarian cancer; ECOG PS, Eastern Cooperative Group performance status; G1, well differentiated; G2, moderately differentiated; G3, poorly differentiated; FIGO, International Federation of Gynecology and Obstetrics; CA -125, cancer antigen 125;LMR, lymphocyte-to-monocyte ratio.

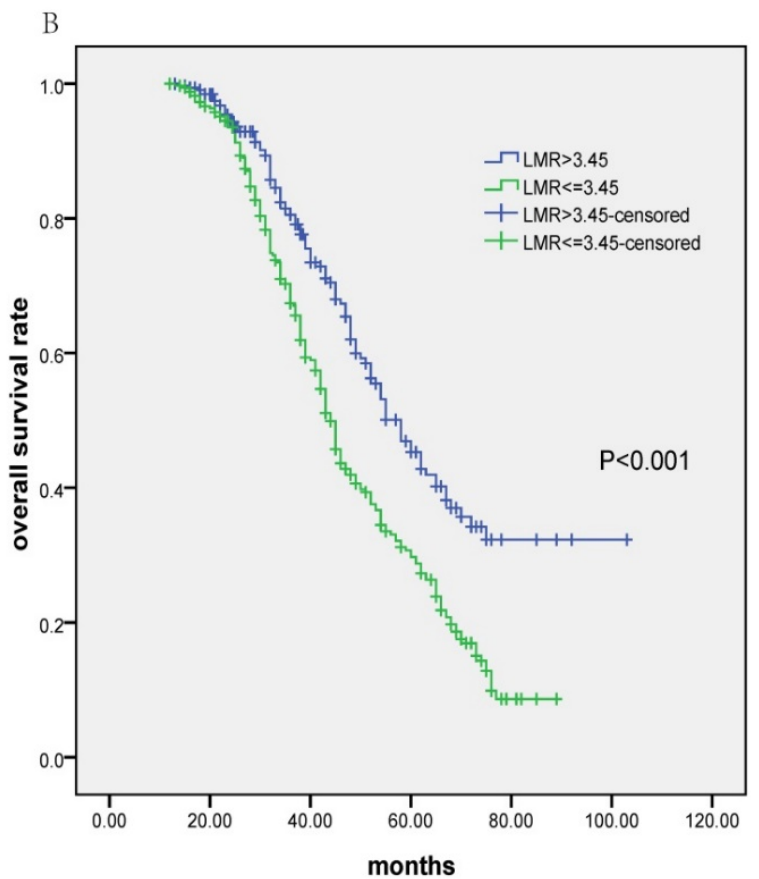

Figure 2. Kaplan-Meier survival analysis of lymphocyte-to-monocyte ratio (LMR) in patients with advanced epithelial ovarian cancer receiving neoadjuvant chemotherapy (NAC) followed by debulking surgery. A: Progression-free survival according to LMR; B: Overall survival according to LMR 
Table 3. Univariate and multivariate analysis of factors associated with Progression-Free Survival and Overall Survival of all patients.

\begin{tabular}{|c|c|c|c|c|c|c|}
\hline \multirow[t]{3}{*}{ Parameters } & \multicolumn{3}{|c|}{ PFS } & \multicolumn{3}{|c|}{ OS } \\
\hline & Univariate analysis & Multivariate analysis & & Univariate analysis & Multivariate analysis & \\
\hline & Pvalue & $\mathrm{HR}(95 \% \mathrm{CI})$ & Pvalue & Pvalue & $\mathrm{HR}(95 \% \mathrm{CI})$ & Pvalue \\
\hline Age $\geq 55$ years & 0.447 & & & 0.298 & & \\
\hline ECOG PS score $(\geq 2)$ & 0.635 & & & 0.369 & & \\
\hline $\begin{array}{l}\text { Histological type } \\
\text { Serous vs. Non-serous }\end{array}$ & 0.039 & & & 0.041 & & \\
\hline $\begin{array}{l}\text { Histological grade } \\
\text { G2/G3 vs. G1 }\end{array}$ & $<0.001$ & & & 0.027 & & \\
\hline $\begin{array}{l}\text { FIGO stage } \\
\text { IV vs. II }\end{array}$ & $<0.001$ & $1.526(1.246-1.869)$ & $<0.001$ & $<0.001$ & 1.541 (1.198-1.982) & 0.001 \\
\hline CA-125 (U/mL ) >35 & 0.031 & & & 0.010 & & \\
\hline $\begin{array}{l}\text { Chemosensitivity } \\
\text { No vs. Yes }\end{array}$ & 0.014 & $1.483(1.004-2.190)$ & 0.048 & 0.002 & 1.317 (1.039-1.669) & 0.023 \\
\hline Residual tumor $(\mathrm{cm})>1$ & 0.026 & $1.287(1.032-1.606)$ & 0.025 & 0.005 & 1.291 (1.014-1.643) & 0.038 \\
\hline $\mathrm{LMR} \leq 3.45$ & $<0.001$ & 1.721 (1.505-1.969) & $<0.001$ & $<0.001$ & 1.625 (1.388-1.895) & $<0.001$ \\
\hline
\end{tabular}

Increasing evidence suggests that the pre-treatment host systemic immune response and inflammatory level can predict patient survival in advanced cancer. LMR may be a sensitive host immunity-related prognostic indicator in cancer patients [18]. Previous investigations in a large cohort study, which included 1547 cases, showed that non-metastatic nasopharyngeal carcinoma patients with higher LMR levels $(\geq 5.22)$ had significantly better PFS and OS [19]. Subsequent multivariate analysis revealed that higher LMR level was still a significant favorable prognostic factor. Lin et al. reported that elevated LMR $(\geq 4.56)$ was associated with better PFS and OS in metastatic non-small-cell lung cancer patients [20]. In this large cohort study, we analyzed peripheral blood LMR in 672 patients with advanced EOC at diagnosis. The median value of LMR was 3.40. Based on the ROC curve analyses, 3.45 was determined to be the best cutoff value for distinguishing between different prognoses. The patients with low LMR tended to have NAC resistance and residual tumor $>1 \mathrm{~cm}$ compared with patients with higher LMR. Not surprisingly, the median PFS and OS in the low LMR group were significantly shorter than those in the high LMR group. Multivariate analysis also showed that low LMR was an independent prognostic factor for PFS and OS. All these data demonstrated that LMR was closely related to NAC response and prognosis in advanced EOC, indicating a significant role for lymphocytes in inhibiting tumor progression and monocytes in promoting the development of disease.

Williams KA et al. reported that CA-125 was positively correlated with monocyte count and inversely correlated with lymphocyte count on 519 women with ovarian cancer [21]. In a multivariate adjusted analysis, high lymphocyte count predicted better survival. A recent study from South Korea reported by Eo et al. evaluated the prognostic significance of LMR in 234 EOC patients receiving primary debulking surgery [16]. Their results showed that LMR cutoff value of 2.07, which was selected through the ROC curve analysis, can categorize the patients into two groups (low LMR and high LMR) with different survival outcomes [16]. Similarly, LMR is a significant independent prognostic factor for OS, although it is not significant for predicting PFS. There may be several reasons for small differences between the results of our study and the study in South Korea. First, for addressing potential biases associated with different treatment modalities, three cycles of NAC combined with debulking surgery was administered to all patients enrolled into our study. Meanwhile, the patients who had been treated with radiotherapy or neoadjuvant chemotherapy were excluded in the Korea study. Second, $41.4 \%$ of patients had stage I-II disease in the Korea study, whereas only EOC patients with stage III-IV were enrolled into our study. From the above results, LMR is a novel prognostic factor for advanced EOC that can be used to categorize patients into different prognostic subgroups, guiding the personalized treatment.

Approximately $70 \%-80 \%$ of all EOC patients develop advanced diseases [1]. Currently, NAC is recommended by the National Comprehensive Cancer Network guidelines for stages IIIc to IV EOC patients, who are not surgical candidates. However, noticeable differences in chemotherapeutic response and survival outcome after NAC therapy has been observed among advanced EOC patients, indicating that advanced EOC is a heterogeneous disorder. Therefore, great advances have been made in searching predictive or prognostic biomarkers to distinguish heterogeneous advanced EOC. The cell immunity mediated by lymphocytes plays a vital role 
in the antitumor process. Lymphocytes can migrate into the tumor microenvironment and evolve into tumor-infiltrating lymphocytes (TILs). TILs, particularly CD8+T cells and CD20+ B cells, accumulate in the tumor microenvironment and are associated with good outcomes [22-24]. As a surrogate marker of strong antitumor immunity, elevated ALC in peripheral blood correlated with good survival outcomes in patients with extranodal natural killer/T-cell lymphoma [10]. On the other hand, previous studies have showed that monocytes produce various cytokines, for instance, interleukin(IL)-6, IL-10 and IL-15, which have been related to poor prognosis in cancer patients [25-27]. Moreover, monocytes can differentiate into tumor-associated macrophage, which promote the proliferation of tumor cells and tumor angiogenesis and enhance the metastatic potential of malignant cells [28]. Low AMC in peripheral blood has been found to be associated with good prognosis in NPC patients [19]. However, either ALC or AMC, as a single clinical parameter, seemed to have limited ability to predict survival outcome. Taking these aspects into consideration, LMR, as an indicator of systemic immunity and inflammation, can be an ideal candidate prediction marker because of the advantages of simplicity and easy availability compared with complex molecular markers.

LMR, as an easily measured clinical biomarker, was proven to be a prognostic stratification factor for advanced EOC patients in our study, which had the advantages of including large sample size, involved multicenter data and low heterogeneity of patients receiving NAC followed by debulking surgery. Meanwhile, limitations of this study include its retrospective design, which can lead to selection bias. Prospective studies are essential to validate our findings in the future.

In conclusion, this is the first large cohort study that confirms LMR is a powerful predictor of NAC and is a prognostic factor in patients with advanced EOC. Future experiment research is needed to further investigate the correlation between LMR and tumor microenvironment in advanced EOC.

\section{Supplementary Material}

Supplementary figure $S 1$.

http://www.jcancer.org/v08p0737s1.pdf

\section{Acknowledgments}

We thank the patients and their families and all the investigators, including the physicians, nurses, and laboratory technicians in this study.

\section{Funding}

This study was funded by Science and Technology Projects of Guangdong Province (contract/grant number: 2014A020212577), Young Teachers' Cultivation Project of Sun Yat-Sen University (contract/grant number: 16ykpy30), Medical Research Foundation of Guangdong Province (contract/grant number: A2015008) and National Natural Science Foundation of China (contract/grant number: 81472469).

\section{Ethical approval}

All procedures performed in studies involving human participants were in accordance with the ethical standards of the institutional and national research committee and with the 1964 Helsinki declaration and its later amendments or comparable ethical standards.

\section{Informed consent}

Informed consent was obtained from all individual participants included in the study.

\section{Competing Interests}

The authors have declared that no competing interest exists.

\section{References}

1. Jelovac D, Armstrong DK. Recent progress in the diagnosis and treatment of ovarian cancer. CA: a cancer journal for clinicians. 2011;61(3):183-203.

2. Ferlay J, Parkin DM, Steliarova-Foucher E. Estimates of cancer incidence and mortality in Europe in 2008. European journal of cancer (Oxford, England : 1990). 2010;46(4):765-81.

3. Cannistra SA. Cancer of the ovary. The New England journal of medicine. 2004;351(24):2519-29.

4. Schwartz PE, Zheng W. Neoadjuvant chemotherapy for advanced ovarian cancer: the role of cytology in pretreatment diagnosis. Gynecologic oncology. 2003;90(3):644-50.

5. Lewin SN, Herzog TJ, Barrena Medel NI, Deutsch I, Burke WM, Sun X et al. Comparative performance of the 2009 international Federation of gynecology and obstetrics' staging system for uterine corpus cancer. Obstetrics and gynecology. 2010;116(5):1141-9.

6. Morrow CP, Bundy BN, Kurman RJ, Creasman WT, Heller P, Homesley HD et al. Relationship between surgical-pathological risk factors and outcome in clinical stage I and II carcinoma of the endometrium: a Gynecologic Oncology Group study. Gynecologic oncology. 1991;40(1):55-65.

7. Suh DH, Kim HS, Chung HH, Kim JW, Park NH, Song YS et al. Pre-operative systemic inflammatory response markers in predicting lymph node metastasis in endometrioid endometrial adenocarcinoma. European journal of obstetrics, gynecology, and reproductive biology. 2012;162(2):206-10.

8. Leibowitz-Amit R, Israel A, Gal M, Atenafu EA, Symon Z, Portnoy O et al. Association between the Absolute Baseline Lymphocyte Count and Response to Neoadjuvant Platinum-based Chemotherapy in Muscle-invasive Bladder Cancer. Clinical oncology (Royal College of Radiologists (Great Britain)). 2016.

9. Rochet NM, Kottschade LA, Grotz TE, Porrata LF, Markovic SN. The prognostic role of the preoperative absolute lymphocyte count and absolute monocyte count in patients with resected advanced melanoma. American journal of clinical oncology. 2015;38(3):252-8.

10. Huang JJ, Jiang WQ, Lin TY, Huang $Y, X u$ RH, Huang $\mathrm{HO}$ et al. Absolute lymphocyte count is a novel prognostic indicator in extranodal natural killer/T-cell lymphoma, nasal type. Annals of oncology : official journal of the European Society for Medical Oncology / ESMO. 2011;22(1):149-55.

11. von Hohenstaufen KA, Conconi A, de Campos $\mathrm{CP}$, Franceschetti $\mathrm{S}$, Bertoni $\mathrm{F}$, Margiotta Casaluci G et al. Prognostic impact of monocyte count at presentation in mantle cell lymphoma. British journal of haematology. 2013;162(4):465-73.

12. Hu S, Zou Z, Li H, Zou G, Li Z, Xu J et al. The Preoperative Peripheral Blood Monocyte Count Is Associated with Liver Metastasis and Overall Survival in Colorectal Cancer Patients. PloS one. 2016;11(6):e0157486. 
13. Kumagai S, Marumo S, Shoji T, Sakuramoto M, Hirai T, Nishimura T et al. Prognostic impact of preoperative monocyte counts in patients with resected lung adenocarcinoma. Lung cancer (Amsterdam, Netherlands). 2014;85(3):457-64

14. Lin ZX, Ruan DY, Li Y, Wu DH, Ma XK, Chen $J$ et al. Lymphocyte-to-monocyte ratio predicts survival of patients with hepatocellular carcinoma after curative resection. World journal of gastroenterology. 2015;21(38):10898-906.

15. Eo WK, Kwon S, Koh SB, Kim MJ, Ji YI, Lee JY et al. The Lymphocyte-Monocyte Ratio Predicts Patient Survival and Aggressiveness of Endometrial Cancer. Journal of Cancer. 2016;7(5):538-45.

16. Eo WK, Chang HJ, Kwon SH, Koh SB, Kim YO, Ji YI et al. The Lymphocyte-Monocyte Ratio Predicts Patient Survival and Aggressiveness of Ovarian Cancer. Journal of Cancer. 2016;7(3):289-96.

17. Therasse P, Arbuck SG, Eisenhauer EA, Wanders J, Kaplan RS, Rubinstein L et al. New guidelines to evaluate the response to treatment in solid tumors. European Organization for Research and Treatment of Cancer, National Cancer Institute of the United States, National Cancer Institute of Canada. Journal of the National Cancer Institute. 2000;92(3):205-16.

18. Roxburgh CS, McMillan DC. Role of systemic inflammatory response in predicting survival in patients with primary operable cancer. Future oncology (London, England). 2010;6(1):149-63.

19. Li J, Jiang R, Liu WS, Liu Q, Xu M, Feng QS et al. A large cohort study reveals the association of elevated peripheral blood lymphocyte-to-monocyte ratio with favorable prognosis in nasopharyngeal carcinoma. PloS one. 2013;8 (12): e83069.

20. Lin GN, Peng JW, Xiao JJ, Liu DY, Xia ZJ. Prognostic impact of circulating monocytes and lymphocyte-to-monocyte ratio on previously untreated metastatic non-small cell lung cancer patients receiving platinum-based doublet. Medical oncology (Northwood, London, England). 2014;31(7):70.

21. Williams KA, Labidi-Galy SI, Terry KL, Vitonis AF, Welch WR, Goodman A, et al. Prognostic significance and predictors of the neutrophil-to-lymphocyte ratio in ovarian cancer. Gynecol Oncol. 2014;132(3):542-50.

22. Nielsen JS, Sahota RA, Milne K, Kost SE, Nesslinger NI, Watson PH et al. CD20+ tumor-infiltrating lymphocytes have an atypical CD27- memory phenotype and together with CD8+ T cells promote favorable prognosis in ovarian cancer. Clinical cancer research : an official journal of the American Association for Cancer Research. 2012;18(12):3281-92.

23. Pruneri G, Gray KP, Vingiani A, Viale G, Curigliano G, Criscitiello C et al. Tumor-infiltrating lymphocytes (TILs) are a powerful prognostic marker in patients with triple-negative breast cancer enrolled in the IBCSG phase III randomized clinical trial 22-00. Breast cancer research and treatment. 2016;158(2):323-31.

24. Li Y, Liang L, Dai W, Cai G, Xu Y, Li X et al. Prognostic impact of programed cell death-1 (PD-1) and PD-ligand 1 (PD-L1) expression in cancer cells and tumor infiltrating lymphocytes in colorectal cancer. Molecular cancer. 2016;15(1):55.

25. Torisu-Itakura $\mathrm{H}$, Lee $J H_{\text {, Huynh }} \mathrm{Y}$, $\mathrm{Ye} X$, Essner $\mathrm{R}$, Morton DL. Monocyte-derived IL-10 expression predicts prognosis of stage IV melanoma patients. Journal of immunotherapy (Hagerstown, Md: 1997). 2007;30(8):831-8.

26. Anand M, Chodda SK, Parikh PM, Nadkarni JS. Abnormal levels of proinflammatory cytokines in serum and monocyte cultures from patients with chronic myeloid leukemia in different stages, and their role in prognosis. Hematological oncology. 1998;16(4):143-54.

27. Ishii $\mathrm{H}$, Takahara $\mathrm{M}$, Nagato $\mathrm{T}$, Kis LL, Nagy $\mathrm{N}$, Kishibe $\mathrm{K}$ et al. Monocytes enhance cell proliferation and LMP1 expression of nasal natural killer/T-cell lymphoma cells by cell contact-dependent interaction through membrane-bound IL-15. International journal of cancer Journal international du cancer. 2012;130(1):48-58.

28. Condeelis J, Pollard JW. Macrophages: obligate partners for tumor cell migration, invasion, and metastasis. Cell. 2006;124(2):263-6. 